\title{
A Research of Truck's Quality Online Estimation Based on CAN Information
}

\author{
Liangkun Yang ${ }^{1,2, *}$, Jie Jin ${ }^{1,2}$, Zhichao Liu ${ }^{1,2}$ and Juanjuan Chen ${ }^{1,2}$ \\ ${ }^{1}$ Transport Vehicles Ministry of Transport Research Institute of Highway Ministry of Transport, Courtyard 8\#, Xitucheng Road, \\ Haidian District, 100088, China \\ ${ }^{2}$ Beijing Zhong Gong Gao Yuan Automotive Test Ltd, 101149, China \\ ${ }^{*}$ Corresponding author
}

\begin{abstract}
The quality of trucks has a huge change from empty to fully load; the change of quality highly affects truck's driving ${ }^{[1]}$. This paper takes a truck as research object and structured the vehicle quality online estimation model considering LS and vehicle sensor technology; the study set the default value of the road gradient to 0 and this can not only satisfy better calculation accuracy, but also enhance the efficiency and instantaneity of the algorithm. We created a quality online estimation model with MATLAB/SIMULINK and a truck dynamics simulation model with TRUCKSIM; after the simulation analysis in different operating conditions, we verified the quality online estimation model ${ }^{[2]}$. It can be found that when the model is running in different gears, there will be large data fluctuations in the initial stage of simulation, and it will quickly converge to the allowable error range of the truth value in 2 minutes. The model can guarantee the 5 percent accuracy and good simulation results.
\end{abstract}

Keywords-CAN; truck; TRUCKSIM; LS; quality online estimation; S-Function

\section{INTRODUCTION}

The change of trucks' loading quality has a great influence on the running status of vehicles. How to realize the online estimation of trucks' loading quality is one of the problems that must be solved in the current vehicle engineering; at present, there are two common methods for the quality inspection of trucks: 1) Direct mass measurement at a fixed location, This method is simple and has a high accuracy, but it cannot perform dynamic detection of the vehicle mass; 2) Measuring leaf spring vertical force through additional installed sensors to achieve dynamic detection of vehicle mass based on the suspension dynamics. This method has a high accuracy and a good instantaneity, but the additional sensors usually cost too much and need lots of workload ${ }^{[3]}$. Therefore, the online statistics of trucks' dynamic information based on the CAN bus have great technical advantages and potential development because of its real-time information transmission and convenient statistical means.

System identification theory is an important technical means to complete the study; at present, this theory is becoming more and more mature, and is widely used in various engineering and technical fields of national economy and national defense construction, which including aerospace, aviation, navigation, robotics, industry and automobile engineering. As a common idea, system identification theory has become the basis for many advanced control technologies such as predictive control, sliding mode variable structure control, intelligent control, neural network control, fuzzy control, robust control, and so on. System identification and adaptive control theory have made a theoretical foundation for this paper's vehicle on-line quality mass estimation ${ }^{[4][5]}$.

According to the characteristics of the large variation in the total loading mass of trucks, this paper developed a method for on-line estimation of the trucks' quality based on CAN information using the system identification theory to achieve real-time dynamic detection of the loading quality of trucks, it also carried on the simulation verification of the on-line quality estimation method and corrected the model based on the vehicle dynamics simulation software. The on-line estimation method of truck's quality based on CAN information can realize the pre-installation of vehicle quality on-line detection device, which has important practical significance for improving the safety and fuel economy of trucks. This paper integrated system identification theory, adaptive control theory, nonlinear dynamics theory, vehicle sensor technology, virtual reality technology and other relevant theories and techniques to study vehicle quality online estimation methods and to solve the problem of vehicle quality's on-line estimation.

\section{SEgMENTED LEAST SQUARES}

The development of system identification theory has been very mature so far, and it is usually applied to many advanced control technologies, and LS is one of the most popular identification methods. It can find the best function matching of data by minimizing the square of the error, and is widely applied to national economy and defense construction because of its simple principle, good stability, and strong robustness ${ }^{[6]}$; however, the least square method has a poor real-time performance in the computing process, so we need to develop a more efficient identification method. There are two methods which are widely used currently; the first one is overall leastsquares method and the other one is segmented least-squares method. In the overall least-squares method, people consider that there are interferences in both of the regression matrix and the observation vector, in the calculation process, the interferences must be considered to obtain more accurate results; However, its application has been limited because this method is more complicated than the least squares method and 
the accuracy of the parameters also needs to be further studied.

The recursive least square method can obtain the identification results which are satisfied the accuracy requirement by the continuously Iteration and correction of the previous estimated value, and it also can save storage space and reduce the amount of calculation because the intermediate amount of calculation does not need to be stored.

Assume that the data input of the SISO system is ${ }^{[7]}$ :

$$
A\left(z^{-1}\right) \text { y }(\mathrm{k})=\mathrm{B}\left(z^{-1}\right) u(k-d)+e(k)
$$

$\mathrm{e}(\mathrm{k})$ is the white noise generated during the calculation, when the objective function satisfies:

$$
H=\delta^{T} \delta=(y-\phi \hat{\theta})^{T}(y-\phi \hat{\theta})
$$

So that we can get one calculation result of $\hat{\theta}$ which is an estimate of the unknown parameter; however, this least-squares method uses a large amount of memory for each calculation in the calculation process, and it cannot be real-time estimated because of its long computation time. If we can use a correction value of $\hat{\theta}(k)$ in the iteration process, that is $\hat{\theta}(k+1)=\hat{\theta}(k)+\gamma$, the correction value is $\gamma$, and then we can get the continuous update of the estimated value; because the intermediate amount of calculation does not need to be stored in the computer, it can also realizes real-time operation of the result.

The recursive least squares recurrence formula is:

$$
\begin{aligned}
& P(k)=P(k-1)-K(k) \Phi^{T}(k) P(k-1) \\
& K(k)=P(k-1) \Phi(k)\left[\Phi^{T}(k) P(k-1) \Phi(k)+1\right] \\
& \hat{\theta}(k)=\hat{\theta}(k-1)+K(k)\left[y(k)-\Phi^{T}(k) \hat{\theta}(k-1)\right]
\end{aligned}
$$

\section{LONGITUDINAL DYNAMIC MODEL OF TRUCKS}

\section{A. Establishment of Longitudinal Dynamic Model}

In this paper, the longitudinal dynamic model of the truck is obtained according to the force relationship when the vehicle is driving (Figure I) and the longitudinal the classical dynamic model of vehicle ${ }^{[8][9]}$. When trucks are driving on slopes, the vibration along the $\mathrm{Y}$-axis will have an important impact on the quality estimation results because of the large slope changes. At present, most of the highway slopes in China are within 5\%. Therefore, this model ignores the impact of slopes on quality estimations in order to complete process quickly and efficiently

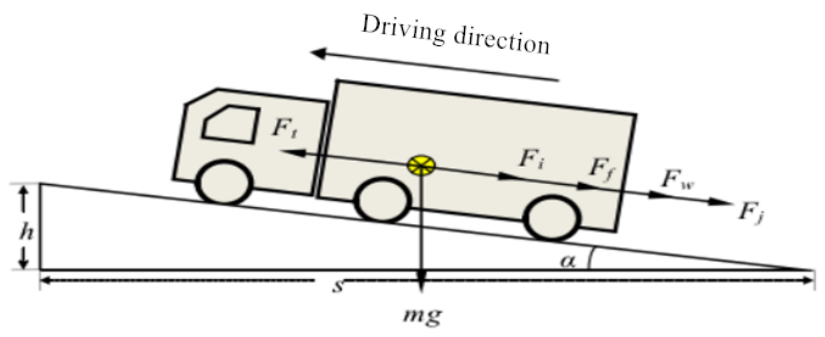

FIGURE I. LONGITUDINAL FORCE DIAGRAM OF TRUCKS WHEN DRIVING

Drive force-Resistance balance equation:

$$
\frac{T_{t q} i_{g} i_{0} \eta_{T}}{R}=M g \sin \alpha+M g f \cos \alpha+\frac{C_{d} A}{21.15} u_{a}^{2}+\delta M \frac{d u}{d t}
$$

Ignore the impact of the road slope on the estimation, which is $\alpha=0$ :

$$
M_{V}=\frac{T_{t q} i_{g} i_{0} \eta_{T}}{R}-M g f-\frac{C_{d} A}{21.15} u_{a}^{2}
$$

In this formula: $\mathrm{M}$ is the quality of the truck; $V$ is the longitudinal acceleration of the truck; ${ }^{T_{q q}}$ is the output torque of the truck, $i_{g} i_{0}$ is the gear ratio of the transmission and the final drive ratio, $R$ is the rotation radius of wheels, $f$ is the road adhesion coefficient, $C_{d}$ is the coefficient of air resistance. $A$ is Windward area of the truck and is the speed of the truck;

After simplifying the above formula, the Drive forceResistance balance equation is obtained:

$$
\begin{aligned}
& F_{R}=\operatorname{Mgf}+\frac{C_{d} A}{21.15} u_{a}^{2} \\
& F_{t}=\frac{T_{t q} i_{g} i_{0} \eta_{T}}{R}
\end{aligned}
$$

$$
F_{t}=M_{V}+F_{R}
$$

If ${ }^{\bar{M}}-\bar{F}_{R}$ is the actual value of the quality and resistance; $M-F_{R}$ is its estimated value, then the above formula can be converted into: 


$$
\left\{\begin{array}{l}
F_{t}=M_{V}+\bar{F}_{R} \\
F_{t}=\bar{M}_{V}+F_{R}
\end{array}\right.
$$

If $\mathrm{m}$ and $\mathrm{n}$ are the sample numbers of the mass and the resistance, it can be got that:

$$
\left\{\begin{array}{l}
\sum_{i=1}^{m} F_{t}=\sum_{i=1}^{m} M V+m \bar{F}_{R} \\
\sum_{j=1}^{n} F_{t}=\sum_{j=1}^{n} \bar{M}_{V}+n F_{R}
\end{array}\right.
$$

Brought the above formula into the Drive force-Resistance balance equation, and we can got that:

$$
\left\{\begin{array}{l}
F=\frac{1}{m} \sum_{i=1}^{m} F_{t, i}-\frac{\bar{M}}{m} \sum_{i=1}^{m} V_{i} \\
M=\frac{\sum_{i=1}^{n} F_{t, i} V_{i}}{\sum_{i=1}^{n} V^{2}}-\frac{\overline{F_{R}} \sum_{i=1}^{n} V_{i}}{\sum_{i=1}^{n} V^{2}}
\end{array}\right.
$$

Make the system sampling frequency consistent with the truck's CAN output frequency, and make quality estimation with the above formula by segmented recursive least squares, so we can get that:

$$
\begin{aligned}
& P(k)=P(k-1)-K(k) \Phi^{T}(k) P(k-1) \\
& K(k)=P(k-1) \Phi(k)\left[\Phi^{T}(k) P(k-1) \Phi(k)+1\right] \\
& \hat{\theta}(k)=\hat{\theta}(k-1)+K(k)\left[y(k)-\Phi^{T}(k) \hat{\theta}(k-1)\right]
\end{aligned}
$$

For the quality estimation in this paper, the meaning of each parameter is:

$$
\left\{\begin{array}{l}
\theta=M, \text { is quality of the truck; } \\
\mathrm{y}=F_{t}-M_{V}, \text { is driving resistance of the truck; } \\
\Phi=v, \text { is acceleration of the truck; }
\end{array}\right.
$$

\section{B. Selection of Vehicle Model in TruckSim}

TruckSim is one vehicle dynamics simulation software; the vehicle model can make response to the inputs of the driver, road surface, and aerodynamic. It is mainly used to predict and simulate the stability and braking power of vehicle, ride of the automobile, power performance and fuel economy of the vehicle. At the same time, it is widely used in the development process of modern automotive control systems; the operating interface is shown in Figure II;

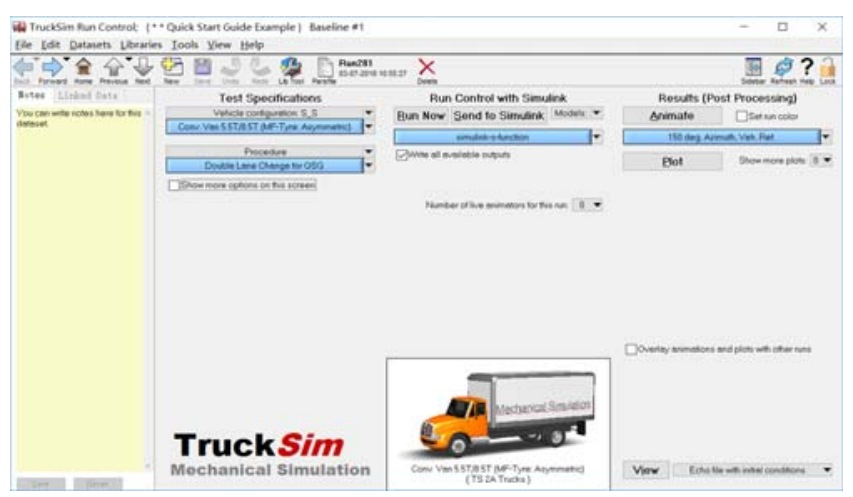

FIGURE II. OPERATING INTERFACE OF TRUCKSIM

TruckSim can export more than 560 variable parameters that can be user-defined. Such as vehicle dimensions, quality, engine power, engine capacity, vehicle position, posture, and

\begin{tabular}{|c|c|c|c|}
\hline $\begin{array}{c}\text { Name of } \\
\text { parameters }\end{array}$ & $\begin{array}{c}\text { Sym } \\
\text { bol }\end{array}$ & Unit & Value \\
\hline Vehicle & $\mathrm{L} * \mathrm{~W}$ & $\mathrm{~mm}$ & $10120 * 2480 * 3895$ \\
\hline dimensions & $*^{H} \mathrm{H}$ & & \\
\hline Curb mass & $\mathrm{M}_{0}$ & $\mathrm{Kg}$ & 5635 \\
\hline Full mass & $\mathrm{M}_{1}$ & $\mathrm{Kg}$ & 12135 \\
\hline Engine power & $\mathrm{P}$ & kw & 305 \\
\hline Engine capacity & V & $\mathrm{ml}$ & 11760 \\
\hline $\begin{array}{l}\text { Coefficient of } \\
\text { air resistance }\end{array}$ & $\mathrm{C}_{\mathrm{D}}$ & -- & 0.7235 \\
\hline Windward area & A & $\mathrm{m}^{2}$ & 7.936 \\
\hline Final drive ratio & $\mathrm{ig}_{\mathrm{g}}$ & -- & 5.276 \\
\hline $\begin{array}{c}\text { Gear ratio of the } \\
\text { transmission }\end{array}$ & $\mathrm{i}_{0}$ & -- & $\begin{array}{l}7.340 / 5.240 / 3.760 / 2.820 / \\
1.950 / 1.390 / 1.000 / 0.750\end{array}$ \\
\hline Efficiency & $\eta_{\mathrm{T}}$ & -- & 0.8912 \\
\hline Rotation radius & $\mathrm{R}$ & $\mathrm{m}$ & 0.5499 \\
\hline
\end{tabular}
movement variables, etc ${ }^{[10]}$. In this experimental process of the model, we use the default parameter inputs of software to simulate the CAN vehicle information inputs and other parameter inputs of the truck, the parameters are in the following table (See Table I):

TABLE I. PARTIAL INPUT PARAMETERS OF MODEL

IV. CO-SIMUlation OPERATION OF MATLAB/TRUCKSiM

The Simulink module in MATLAB is an interactive tool for modelling, simulating and analysing dynamic multidimensional systems; it can complete the model building by using the standard modules in Simulink and can also freely build a variety of model libraries to complete the system description, simulation evaluation and other operations. In this paper, it 
uses the existing vehicle model parameters of TruckSim software as the outputs to the Simulink module, and then uses the S-Function of Simulink module to build the model of the segmented recursive least square method ${ }^{[11]}$. S-Function is an extended Simulink performance tool which can perform function calls by defining the module's state vectors, inputs, task indications, and block parameters, the S-Function block diagram of this model is shown in Figure III.

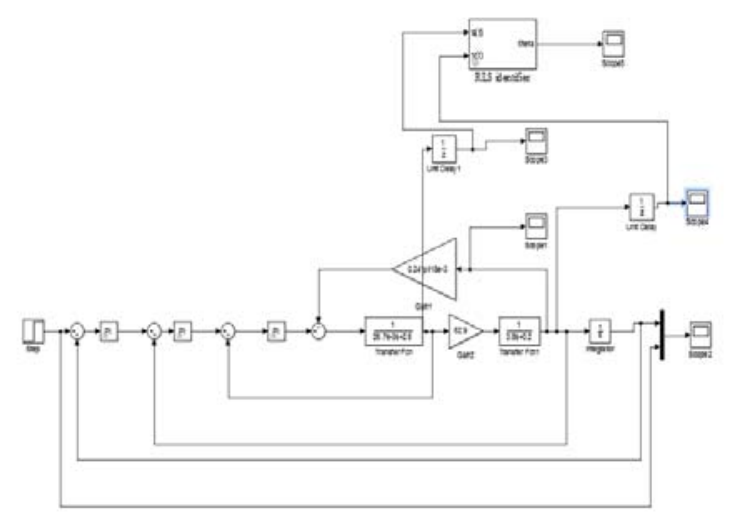

FIGURE III. BLOCK DIAGRAM OF S-FUNCTION

\section{Simulation}

\section{A. Simulation Results of Quality Estimation in Different} Gears

Select the top-gear and full-loading test data to verify the simulation. Figures IV to V show the simulated quality and actual quality comparison values for multiple simulations at different gears. The real quality of the mass identification model is 12.13t; the identification result shows that the peak value of the estimated value can reach 14-15t before stability, and the error is over $20 \%$. However, the estimation can gradually converge to the true value after $2 \mathrm{~s}$ and remain stable with the error within $5 \%$.

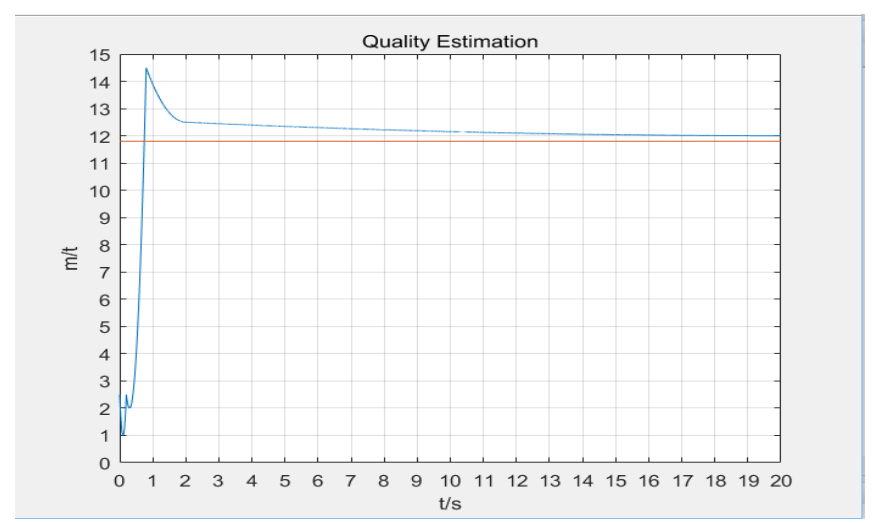

FIGURE IV. TOP-GEAR SIMULATION VERIFICATION

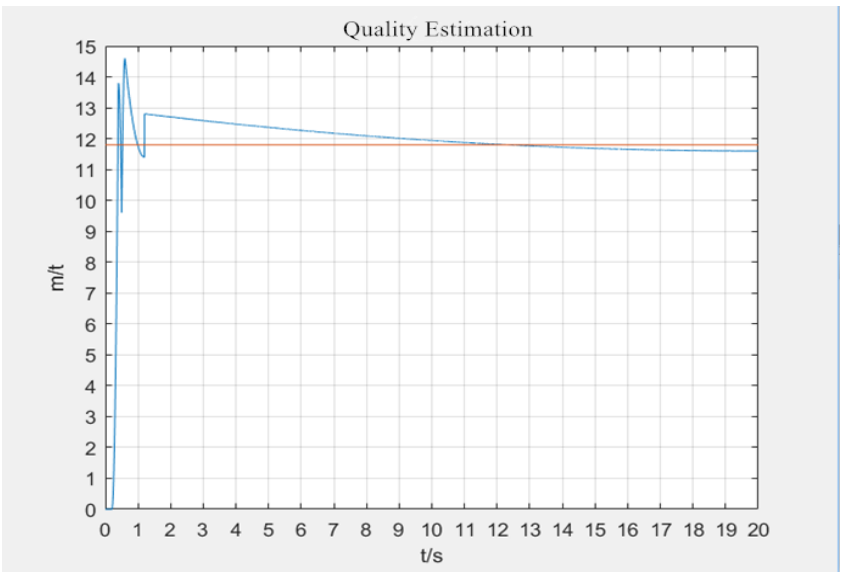

FIGURE V. SEVENTH-GEAR SIMULATION VERIFICATION

It can be seen from Figure IV and Figure $\mathrm{V}$ that the effect of parameter changes such as the gears of the truck on the quality estimation is only at the initial stage of the estimation, there will be large fluctuations at this stage; and the trend of changes in the curve is about the same after the stabilization. The quality estimation at top-gear reached the peak value of $14.32 \mathrm{t}$ at $0.9 \mathrm{~s}$ and remained stable with the error within $5 \%$ in $1.9 \mathrm{~s}$; the quality of the seventh gear reached around the true value in $2.2 \mathrm{~s}$. The results are shown in Table II.

TABLE II. DIFFERENT GEAR QUALITY IDENTIFICATION RESULTS

\begin{tabular}{cccc}
\hline Gear & Real quality/t & $\begin{array}{c}\text { Estimated } \\
\text { quality /t }\end{array}$ & Error/\% \\
\hline VIII & 11.86 & 12.13 & 2.3 \\
VII & 11.86 & 11.67 & -1.6 \\
\hline
\end{tabular}

After all, this model has a certain influence on the results only at the beginning of the identification in different gears. After that, the quality estimation can be reached to the real value in a short time, which has a good instantaneity and accuracy.

\section{B. Simulation Results of Quality Estimation in Different Starting Acceleration}

It can be seen from the quality estimation model that the longitudinal acceleration of the vehicle is an important input parameter, and different acceleration inputs have a certain influence on the instantaneity and accuracy of the quality identification; in this paper, the input values of the vehicle longitudinal acceleration are $V_{1}=0.45 \mathrm{~m} / \mathrm{s}^{2}$ and $V_{2}=0.15 \mathrm{~m} / \mathrm{s}^{2}$, and the corresponding simulation results are shown in Figure VI and Figure VII. 


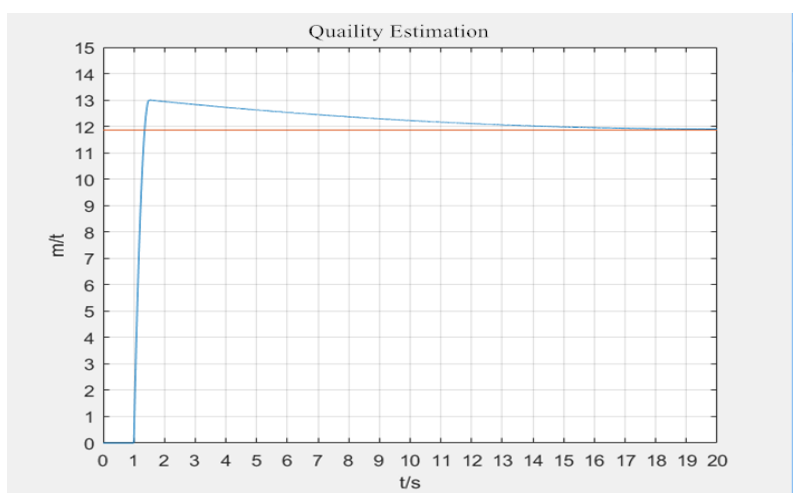

FIGURE VI. ESTIMATION RESULTS AS V1 $=0.45 \mathrm{M} / \mathrm{S} 2$

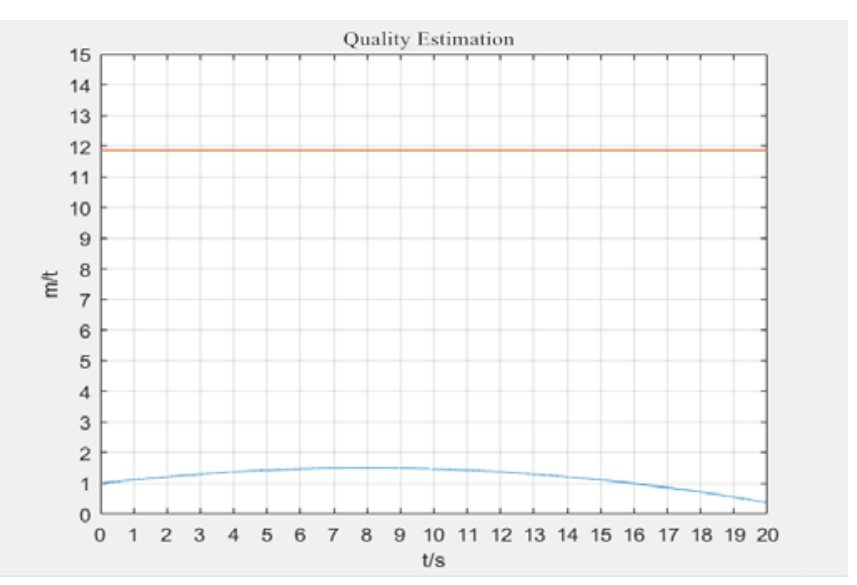

FIGURE VII. ESTIMATION RESULTS AS V2=0.15M/S2

From the above figures, we can see that the different longitudinal accelerations of the vehicle has a greater effect on the quality estimation compared to the effect of the gears on the quality estimation process, When the longitudinal acceleration is less than a certain threshold, the algorithm cannot even recognize the quality model; but in the actual driving conditions, the longitudinal acceleration of the vehicle is usually above $0.3 \mathrm{~m} / \mathrm{s} 2$, Therefore, the impact on the actual quality identification process is small; therefore, it has a small effect on the actual quality estimation process; The results of the different starting acceleration masses are shown in Table III. The data in the table and the analysis of this paper show that the model has high accuracy and good practical application value.

\begin{tabular}{cccc}
\multicolumn{4}{c}{ TABLE III. QUALITY ESTIMATION RESULTS WITH DIFFERENT } \\
STARTING ACCELERATION \\
\hline Accelerations/m/s $\mathbf{s}^{\mathbf{2}}$ & $\begin{array}{c}\text { Real } \\
\text { quality/t }\end{array}$ & $\begin{array}{c}\text { Estimated } \\
\text { quality/t }\end{array}$ & Error/\% \\
\hline 0.45 & 11.86 & 12.01 & 1.3 \\
0.15 & - & - & - \\
\hline
\end{tabular}

\section{CONCLUSION}

This paper uses MATLAB/Simulink module and TRUCKSIM software to construct the real-time quality estimation model based on the input of CAN information of the truck by comprehensively considering the system identification theory and vehicle sensing technology; it can provide a frontier technical support for the real-time supervision of the truck quality and the truck turnover. The model built in this paper regards quality as a slowly variable parameter, so the model has good robustness. At the same time, we can see from the simulation results that for different gears and starting accelerations, there will be fluctuations in the initial stage of simulation; however, the quality estimation can be stable within $2 \mathrm{~s}$. In this paper, the model will take a long time to converge to the real-value under the condition of nonsustained excitation; it mainly considers the driving conditions of straight highways and ignores the slope impact, so that the accuracy under large slope conditions needs to be further verified. In future studies, we can introduce more complex conditions to improve the applicability and accuracy of the algorithm.

\section{REFERENCES}

[1] Jiang Xiaozu. Vehicle Dynamics Modelling and Simulation Analysis of Heavy-duty Truck based on TruckSim[D].Changchun: Jilin University, 2009.

[2] Yang Xiujian, Kang Nan. Tractor-Semitrailer Stability Control Based on TruckSim-Simulink Co-simulation. [J].Journal of Highway and Transportation Research and Development, 2013,30 (3).

[3] Tian Jingjing, Li Shiwu, Su Jian, Wang Linghong. Dynamic monitoring and early-warning system for overload of truck based on displacement sensor [J].Journal of Jilin University(Engineering and Technology Edition), 2012, 42 (6).

[4] Qiang Minghui, Zhang Jinge. Recursive Least Squares Identification and Simulation Based on MATLAB [J]. AUTOMATION \& INSTRUMENTATION, 2008, 06.

[5] Yang Haiqing, Yang Xiujian, Chen Shuqiao, Gao Jin. Research on Coestimation Method of Mass and Grade for Heavy Vehicles [J]. Automobile Technology, 2015,08.

[6] Li Yuanfang. Research on the Identification of Mass for Heavy Vehicle and the Estimation of Road Gradient[D].Chang Chun; Jilin University,2012.

[7] Lin Fen, Huang Chao, etc. Serial Two-parameter Identification of Vehicles Based on RLS [J]. Journal of South China University of Technology (Natural Science Edition), 2012,40(12).

[8] Yu Zhisheng. Automobile Theory [M].Bei Jing: China Machine Press, 2006

[9] Wang Wangyu. Automobile Design [M]. Bei Jing: China Machine Press, 2004.

[10] A.Vahidi, A.Stefanopoulou \& H.Peng. Recursive least squares with forgetting for online estimation of vehicle mass and road grade: theory and experiments [J]. Vehicle System Dynamics, 2005,43(01)

[11] Ardalan Vahidi, Anna Stefanopoulou. Experiments for Online Estimation of Heavy Vehicle's Mass and Time-Varying Road Grade [J]. University of Michigan, 2002. 